\title{
Nuevo coronavirus (COVID-19). Un análisis bibliométrico
}

\section{Coronavirus disease (COVID-19). A bibliometric analysis}

Nicolás O’Brien ${ }^{1}$, Mg. Miguel Barboza-Palomino², José Ventura-León², Mg. Tomás Caycho-Rodríguez², José S. Sandoval-Díaz³, Wilson López-López ${ }^{4}$, Gonzalo Salas ${ }^{5}$

\begin{abstract}
Objective: The new coronavirus pandemic (COVID-19) has spread to 180 countries, causing more than half a million confirmed cases and more than 40 thousand deaths. The main objective of this study was to analyze the world scientific production related to COVID-19. Material and Method: A literature search was performed using the Scopus database. A total of 547 published documents were identified, analyzing characteristics such as: type of publication, collaboration index, most productive countries, scientific journals, the institutions that are publishing the most on the subject and indicators of citations and impact. Results: China was ranked first with 213 published documents (20.9\%). Regarding the journals with the higher number of published documents, these were The Lancet, British Medical Journal and the Journal of Medical Virology. Likewise, Wuhan University was identified as the institution presenting the highest leadership regarding the number of publications. The total number of citations for all the publications was of 1.685 . The h-index of the retrieved articles was of 42, and four articles were found with more than 100 quotations. Conclusions: This study offers a first approach to the global efforts aimed to this new area of research, which in only three months has substantially increased, and is expected to remain being prolific.
\end{abstract}

\section{Key words:}

Coronavirus, COVID-2019, 2019-nCoV, SARS-COV-2, bibliometrics

\footnotetext{
${ }^{1}$ Cátedra de Anestesiología y Reanimación, Facultad de Medicina, Universidad de Valparaíso, Chile.

${ }^{2}$ Universidad Privada del Norte. Lima, Perú.

${ }^{3}$ Universidad del Bío-Bío. Chillán, Chile.

${ }^{4}$ Pontificia Universidad Javeriana. Bogotá, Colombia.

${ }^{5}$ Departamento de Psicología, Universidad Católica del Maule. Talca, Chile.
}

Fecha de recepción: 30 de marzo de 2020

Fecha de aceptación: 1 de abril de 2020

\section{ORCID}

https://orcid.org/0000-0003-0707-8188

Correspondencia:

Gonzalo Salas

E-mail: gsalas@ucm.cl 


\section{RESUMEN}

Objetivo: La pandemia del nuevo coronavirus (COVID-19) se ha extendido a 180 países, ocasionando más de medio millón de casos confirmados y más de 40.000 muertes. El objetivo principal de este estudio fue analizar la producción científica mundial relacionada con el COVID-19. Material y Métodos: Se realizó una búsqueda bibliográfica utilizando la base de datos Scopus. Se identificaron un total de 547 documentos publicados, analizándose características tales como: modalidad de publicación, los índices de colaboración, los países más productivos, las revistas científicas, las instituciones que más están publicando sobre el tema e indicadores de citación e impacto. Resultados: Se identifica a China como el país que ocupa el primer lugar con $213(20,9 \%)$ documentos publicados. Por su parte, The Lancet, British Medical Journal y el Journal of Medical Virology son las revistas con mayor número de documentos publicados. A su vez, se identifica a la Wuhan University como la institución que presenta mayor liderazgo respecto al número de publicaciones. El número total de citas de todas las publicaciones fue de 1.685 , el índice $h$ de los artículos recuperados fue de 42 y se ubican cuatro artículos con más de 100 citas. Conclusiones: El presente estudio ofrece una primera aproximación a los esfuerzos globales dirigidos a esta nueva área de investigación, que en solo tres meses se ha incrementado de forma sustancial y que se estima continúe siendo prolífica.

\section{Palabras clave:}

Coronavirus, COVID-2019, 2019-nCoV, SARS-CoV-2, bibliometría

\section{Introducción}

E COVID-19 es una enfermedad viral respiratoria causada por un nuevo coronavirus llamado SARS-CoV-2[1]. Desde el brote del síndrome respiratorio agudo severo (SARS) hace 18 años, se han descubierto un gran número de coronavirus relacionados con el SARS (SARSr-CoV), siendo su huésped natural los murciélagos[2],[3]. Los infectados por el COVID-19 pueden desarrollar síntomas leves como tos seca, dolor de garganta y fiebre; mientras que otros pueden llegar a desarrollar condiciones mortales como el shock séptico, edema pulmonar, neumonía severa y el síndrome de dificultad respiratoria aguda [4]. Lamentablemente, hasta la fecha no se conoce un tratamiento farmacológico efectivo y comprobado contra el COVID-19[5].

Este nuevo brote se notificó por primera vez en Wuhan (China) el 12 de diciembre de 2019[6] vinculado con un mercado mayorista de mariscos que comercializaba pescado, aves, murciélagos, serpientes, y otras especies de animales vivos[7]-[9]. Desde entonces se han notificado decenas de miles de casos y el brote se ha extendido a nivel mundial[10]-[12], por lo cual la OMS la declaró pandemia[13],[14]. Hasta el
31 de marzo de 2020 se habían notificado 857.487 casos confirmados, 178.034 casos recuperados y 42.107 muertes en el mundo por esta enfermedad. La tasa de detección de casos varía día a día y puede ser monitoreada en tiempo real a través del sitio web de la Johns Hopkins University (URL = https://coronavirus.jhu.edu/map.html) y otros medios[15].

El rápido incremento de casos confirmados y muertes ha generado gran preocupación y representan una fuerte amenaza para la salud pública mundial[16]. Por lo tanto, las acciones de prevención y el control del COVID-19 son extremadamente importantes[17]. Esto también exige que se realice un monitoreo riguroso y continuo de la evidencia científica sobre el COVID-19 para identificar con precisión y predecir la adaptación, evolución, transmisión y patogenicidad de la enfermedad[4]. Aunque se han realizado algunos estudios vinculados a tendencias en relación al COVID-19[18]-[20], hasta la fecha de envío del presente artículo y en una amplia revisión de la literatura disponible no se encuentran estudios bibliométricos que exploren los factores asociados con la actividad de la investigación en el campo. Por lo tanto, el objetivo de este estudio fue analizar la producción científica mundial relacionada con el COVID-19. 


\section{Método}

Para la búsqueda e identificación de los documentos se utilizó la base de datos Scopus, propiedad de Elsevier[21]. Este proceso se realizó el 26 de marzo de 2020 mediante el siguiente algoritmo de búsqueda: [2019-nCoV] OR [COVID-19] OR [SARS-CoV-2] vinculado a título del artículo, resumen y palabras claves.

Inicialmente, se encontraron 551 resultados y se registraron los siguientes tipos de documentos: artículos, editoriales, notas, cartas al editor, revisiones y comunicaciones breves. Se excluyeron publicaciones denominadas fe de erratas y las que no estaban relacionadas con los tópicos propuestos. Es así como la muestra final incluyó 547 documentos, los cuales se dividieron en 257 (46,98\%) artículos originales, 83 $(15,17 \%)$ cartas al editor, $76(13,89 \%)$ editoriales, 75 $(13,71 \%)$ notas, $50(9,14 \%)$ revisiones y $6(1,1 \%)$ comunicaciones breves.

Con los documentos extraídos se organizó en Microsoft Excel una database que incluyó los siguientes datos: nombre de los autores firmantes, título de la publicación, tipo de publicación, año de la publicación, tipo de acceso a la publicación, instituciones de filiación de los autores firmantes, revista de publicación, país de edición y número de citas recibidas.

Esta información permitió en un primer momento realizar un análisis descriptivo e identificar a los países, las revistas y las instituciones que están publicando más sobre el tema. En un segundo momento, considerando al total de autores $(n=3.232)$, se estimaron los índices de colaboración de Lawani[22] y Subramanyam[23]. Después, se identificaron a las publicaciones más citadas y se estimó la media de las citas. Finalmente, con apoyo del software VoSviewer[24] se elaboró una red con los principales ejes temáticos asociados a las palabras claves de las publicaciones.

\section{Resultados}

Los documentos fueron publicados por autores que firmaron con instituciones de filiación provenientes de 69 países y la mayoría de las publicaciones, en total $465(85,01 \%)$ son de acceso abierto. Como era de esperar, China se clasificó en primer lugar en la cantidad de documentos publicados respecto al COVID-19 con 213 publicaciones y el 20,9\% de la producción mundial. A China le siguió los Estados Unidos con 103, Reino Unido con 53 e Italia con 31. También destacan Canadá, Francia y Hong Kong con 26 documentos publicados por país. La lista de los primeros 10 países se puede visualizar en la Tabla 1.
La Tabla 2 muestra las 10 principales revistas en las que se publicaron artículos relacionados con el COVID-19. Cincuenta y cuatro documentos $(9,87 \%)$ fueron publicados en The Lancet, mientras que 43 (7,86\%) fueron publicados en British Medical Journal y $38(6,95 \%)$ en el Journal of Medical Virology. De este ranking, cinco tienen un índice $h>100$. Ocho de las diez revistas se encuentran en el cuartil 1 de Scopus, con excepción del Journal of Medical Virology y el Journal of Microbiology, Inmunology and Infection que se encuentran en el cuartil 2. Es importante destacar que cuatro de las diez revistas más productivas son publicadas en el Reino Unido. Asimismo, de acuerdo con Scopus, la mayoría de las revistas que han publicado sobre el tema se encuentran clasificadas en las áreas de Medicina, Inmunología y Microbiología, y Bioquímica, Genética y Biología Molecular.

Por otra parte, la amplia mayoría de las diez primeras instituciones con mayor cantidad de documentos, fueron publicados en China, siendo la Wuhan University la institución con mayor número de artículos (30). Le sigue la The University of Hong Kong (19) y la Chinese Academy of Sciences (16). Alcanzan a estar en este listado el London School of Hygiene \& Tropical Medicine de Reino Unido y la Aix Marseille University de Francia con 11 publicaciones cada una (Tabla 3).

En cuanto a la colaboración entre los autores firmantes, se obtuvo un índice de Lawani de 5,91 y un índice de Subramanyam de 0,76. El primer índice indica la media ponderada de autores que firman un artículo[22], observándose que en promedio fir-

Tabla 1. Top ten de países más productivos en la temática de COVID-19

\begin{tabular}{rlr}
\hline SCR & Revista & TP \\
$1^{\text {st }}$ & China & 213 \\
$2^{\text {nd }}$ & Estados Unidos & 103 \\
$3^{\text {rd }}$ & Reino Unido & 53 \\
$4^{\text {th }}$ & Italia & 31 \\
$5^{\text {th }}$ & Canadá & 26 \\
$6^{\text {th }}$ & Francia & 26 \\
$7^{\text {th }}$ & Hong Kong & 26 \\
$8^{\text {th }}$ & Alemania & 24 \\
$9^{\text {th }}$ & Taiwán & 20 \\
$10^{\text {th }}$ & Suiza & 19 \\
\hline
\end{tabular}

SCR: Standart Competition Ranking; TP: número total de documentos. 
Tabla 2. Top ten de revistas científicas más productivas en la temática de COVID-19

\begin{tabular}{clccccc}
\hline SCR & Revista & TP & CS2018 & Q & H & País \\
$1^{\text {st }}$ & The Lancet & 54 & 10,28 & Q1 & 700 & Reino Unido \\
$2^{\text {nd }}$ & British Medical Journal & 43 & 1,78 & Q1 & 392 & Reino Unido \\
$3^{\text {rd }}$ & Journal of Medical Virology & 38 & 1,94 & Q2 & 105 & Estados Unidos \\
$4^{\text {th }}$ & Eurosurveillance & 22 & 5,05 & Q1 & 90 & Francia \\
$5^{\text {th }}$ & JAMA. Journal of the American Medical Association & 16 & 6,98 & Q1 & 622 & Estados Unidos \\
$6^{\text {th }}$ & Travel Medicine and Infectious Disease & 13 & 1,80 & Q1 & 37 & Países Bajos \\
$6^{\text {th }}$ & Emerging Microbes and Infections & 13 & 4,36 & $\mathrm{Q} 1$ & 31 & Reino Unido \\
$7^{\text {th }}$ & Intensive Care Medicine & 10 & 4,19 & $\mathrm{Q} 1$ & 176 & Alemania \\
$8^{\text {th }}$ & Journal of Microbiology, Inmunology and Infection & 9 & 1,86 & $\mathrm{Q} 2$ & 48 & Taiwán \\
$9^{\text {th }}$ & New England Journal of Medicine & 8 & 1,80 & $\mathrm{Q} 1$ & 37 & Reino Unido \\
$9^{\text {th }}$ & International Journal of Infectious Diseases & 8 & 2,89 & $\mathrm{Q} 1$ & 73 & Países Bajos \\
\hline
\end{tabular}

SCR: Standart Competition Ranking; TP: número total de documentos; CS: Cite Score 2018; Q: Quartil, H H-Index.

\begin{tabular}{cllc}
\multicolumn{1}{c}{ Tabla 3. Top ten de instituciones más productivas en la temática de COVID-19 } \\
\hline SCR & Institución & TP & País China \\
$1^{\text {st }}$ & Wuhan University & 30 & China \\
$2^{\text {nd }}$ & The University of Hong Kong & 19 & China \\
$3^{\text {rd }}$ & Chinese Academy of Sciences & 16 & China \\
$3^{\text {rd }}$ & Huazhong. University of Science and Technology & 16 & China \\
$3^{\text {rd }}$ & Ningbo University & 16 & China \\
$6^{\text {th }}$ & Chinese Academy of Medical Sciences \& Peking Union Medical College & 15 & China \\
$6^{\text {th }}$ & Tongji Medical College & 15 & China \\
$8^{\text {th }}$ & Capital Medical School & 14 & China \\
$9^{\text {th }}$ & Fudan Univesity & 11 & China \\
$10^{\text {th }}$ & Chinesse University of Hong Kong & 11 & Reino Unido \\
$10^{\text {th }}$ & London School of Hygiene \& Tropical Medicine & 11 & Francia \\
$10^{\text {th }}$ & Aix Marseille University & & 12 \\
\hline
\end{tabular}

SCR: Standart Competition Ranking. TP: Número total de documentos.

maron 6 autores los 547 documentos analizados. Por su parte, el segundo índice señala la proporción de publicaciones con autoría múltiple, indicando los valores próximos a 1 que la mayoría de trabajos fueron publicados por dos o más autores[23]. Si bien, 413 (75,5\%) documentos tuvieron 2 o más autores, tam- bién se identificaron 134 (24,5\%) publicaciones con un autor firmante, las que se relacionan fundamentalmente con editoriales y cartas al editor.

El número total de citas de todas las publicaciones fue 1.685 y una media de 3.08 citas. El índice $\mathrm{h}$ de los artículos recuperados fue de 42 (es decir, 20 


\begin{tabular}{|c|c|c|c|c|c|}
\hline SCR & Autores & Título & Revista & Citas & $\begin{array}{c}\text { Tipo de } \\
\text { documento }\end{array}$ \\
\hline $1^{\text {st }}$ & $\begin{array}{l}\text { Huang } \\
\text { et al.[25] }\end{array}$ & $\begin{array}{l}\text { Clinical features of patients infected with } 2019 \\
\text { novel coronavirus in Wuhan, China }\end{array}$ & The Lancet & 202 & Artículo \\
\hline $2^{\text {nd }}$ & $\begin{array}{l}\text { Zhu } \\
\text { et al.[7] }\end{array}$ & $\begin{array}{l}\text { A novel coronavirus from patients with pneumonia } \\
\text { in China, } 2019\end{array}$ & $\begin{array}{l}\text { New England } \\
\text { Journal of Medicine }\end{array}$ & 139 & Artículo \\
\hline $3^{\text {rd }}$ & $\begin{array}{l}\text { Chan } \\
\text { et al.[26] }\end{array}$ & $\begin{array}{l}\text { A familial cluster of pneumonia associated with } \\
\text { the } 2019 \text { novel coronavirus indicating person-to- } \\
\text { person transmission: a study of a family cluster }\end{array}$ & The Lancet & 113 & Artículo \\
\hline $4^{\text {th }}$ & $\begin{array}{l}\text { Chen } \\
\text { et al.[8] }\end{array}$ & $\begin{array}{l}\text { Epidemiological and clinical characteristics of } 99 \\
\text { cases of } 2019 \text { novel coronavirus pneumonia in } \\
\text { Wuhan, China: a descriptive study }\end{array}$ & The Lancet & 107 & Artículo \\
\hline $5^{\text {th }}$ & $\begin{array}{l}\text { Zhou } \\
\text { et al.[2] }\end{array}$ & $\begin{array}{l}\text { A pneumonia outbreak associated with a new } \\
\text { coronavirus of probable bat origin }\end{array}$ & Nature & 84 & Artículo \\
\hline $5^{\text {th }}$ & $\begin{array}{l}\text { Wang } \\
\text { et al.[27] }\end{array}$ & $\begin{array}{l}\text { Clinical Characteristics of } 138 \text { Hospitalized Patients } \\
\text { with } 2019 \text { Novel Coronavirus-Infected Pneumonia } \\
\text { in Wuhan, China }\end{array}$ & $J A M A$ & 84 & Artículo \\
\hline $7^{\text {th }}$ & $\begin{array}{l}\text { Lu } \\
\text { et al.[6] }\end{array}$ & $\begin{array}{l}\text { Genomic characterisation and epidemiology of } \\
2019 \text { novel coronavirus: implications for virus ori- } \\
\text { gins and receptor binding }\end{array}$ & The Lancet & 73 & Artículo \\
\hline $8^{\text {th }}$ & $\begin{array}{l}\text { Holshue } \\
\text { et al.[28] }\end{array}$ & $\begin{array}{l}\text { First case of } 2019 \text { novel coronavirus in the United } \\
\text { States }\end{array}$ & $\begin{array}{l}\text { New England } \\
\text { Journal of Medicine }\end{array}$ & 53 & Artículo \\
\hline $9^{\text {th }}$ & $\begin{array}{l}\text { Rothe } \\
\text { et al[29] }\end{array}$ & $\begin{array}{l}\text { Transmission of 2019-NCOV infection from an } \\
\text { asymptomatic contact in Germany }\end{array}$ & $\begin{array}{l}\text { New England } \\
\text { Journal of Medicine }\end{array}$ & 51 & Artículo \\
\hline $10^{\text {th }}$ & $\begin{array}{l}\text { Hui } \\
\text { et al.[30] }\end{array}$ & $\begin{array}{l}\text { The continuing 2019-nCoV epidemic threat of } \\
\text { novel coronaviruses to global health - The latest } \\
2019 \text { novel coronavirus outbreak in Wuhan, China }\end{array}$ & $\begin{array}{l}\text { International } \\
\text { Journal of } \\
\text { Infectious Diseases }\end{array}$ & 43 & Editorial \\
\hline
\end{tabular}

artículos han sido citados al menos 20 veces). Existen cuatro artículos con más de 100 citas. El artículo más citado se denomina "Clinical features of patiens infected with 2019 novel coronavirus in Wuhan, China" y tiene 202 citas. Siete de los 10 artículos más citados fueron publicados en The Lancet y New England Journal of Medicine, ambas revistas de Reino Unido que ostentan 4 y 3 artículos de esta lista respectivamente. Los artículos más citados se pueden visualizar en la Tabla 4.

La Figura 1 muestra las principales palabras claves asociadas a la investigación en COVID-19. Se puede apreciar tres clusters representados por tres colores distintos que agrupan las diversas relaciones de los conceptos y en los cuales se pueden visualizar las redes de cocitación. El tamaño de los nodos, sumado a lo central y periférico de estos, permiten apreciar los nexos de un concepto con otro.

\section{Conclusiones}

Las principales conclusiones de este artículo se resumen en los siguientes puntos; 1) China es el país con mayor cantidad de documentos publicados sobre COVID-19; 2) La mayor cantidad de documentos publicados sobre esta materia se publica principalmente en revistas británicas, entre las cuales destacan The Lancet y British Medical Journal. Ocho de las diez revistas científicas con mayor número de documentos publicados se ubican en el cuartil 1 (Q1); 3) Las citas de los documentos más citados corresponden fundamentalmente a artículos científicos. El artículo con mayor número de citas se denomina "Clinical features of patients infected with 2019 novel coronavirus in Wuhan, China" y fue publicado por Huang et al.[25] en The Lancet; 4) La institución con mayor número de documentos publicados es la Wuhan University; 5) Las 


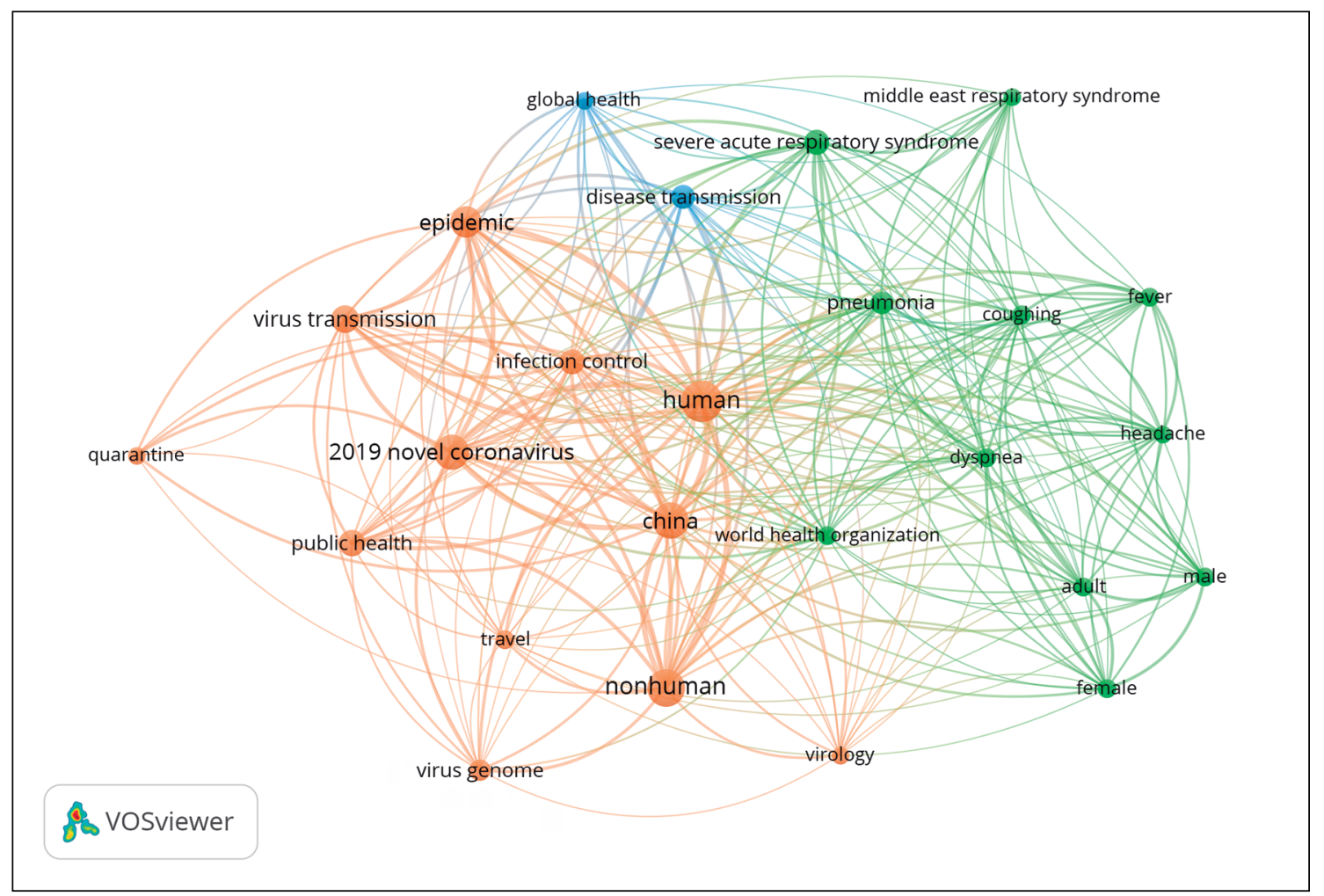

Figura 1. Principales ejes temáticos vinculados a COVID-19.

relaciones analizadas por medio de las palabras clave muestran como sigue siendo un reto dar cuenta de las interdisciplinariedades en un campo concentrado de conocimiento; 6) Es importante hipotetizar que la liberación de un importante número de publicaciones en acceso abierto fue posible debido a la presión sobre las casas editoriales debido a la condición de pandemia; 7) Finalmente, es importante destacar que la rápida evolución de la pandemia COVID-19, las variaciones estadísticas, y la acelerada producción de nuevos resultados de investigación representan una limitación de la presente revisión. Será interesante en futuros estudios realizar este análisis en otras databases como Web of Science y PubMed.

\section{Referencias}

1. Phan T. Genetic diversity and evolution of SARS-

CoV-2. Infect Genet Evol. 2020 Feb;81:104260. https://doi.org/10.1016/j. meegid.2020.104260 PMID:32092483

2. Zhou $P$, Yang $X L$, Wang $X G, H u$ $B$, Zhang $L$, Zhang $W$, et al. A pneumonia outbreak associated with a new coronavirus of probable bat origin. Nature. 2020 Mar;579(7798):270-3. https://doi.org/10.1038/s41586020-2012-7 PMID:32015507

3. Li W, Shi Z, Yu M, Ren W, Smith C, Epstein JH, et al. Bats are natural reservoirs of SARSlike coronaviruses. Science. 2005 Oct;310(5748):676-9. https://doi.org/10.1126/

science. 1118391

PMID:16195424

4. Sohrabi C, Alsafi Z, O'Neill N, Khan M, Kerwan A, AlJabir A, et al. World Health Organization declares global emergency: A review of the 2019 novel coronavirus (COVID-19). Int J Surg. 2020 Apr;76:71-6. https://doi. 
org/10.1016/j.jjsu.2020.02.034

PMID:32112977

5. Cortegiani A, Ingoglia G, Ippolito M, Giarratano A, Einav S. A systematic review on the efficacy and safety of chloroquine for the treatment of COVID-19. J Crit Care. Forthcoming 2020 Mar;S0883-9441(20)30390-7. https://doi.org/10.1016/j. jcrc.2020.03.005 PMID:32173110

6. Lu R, Zhao X, Li J, Niu $\mathrm{P}$, Yang B, Wu $\mathrm{H}$, et al. Genomic characterisation and epidemiology of 2019 novel coronavirus: implications for virus origins and receptor binding. Lancet. 2020 Feb;395(10224):565-74. https://doi.org/10.1016/ S0140-6736(20)30251-8 PMID:32007145

7. Zhu N, Zhang D, Wang W, Li $X$, Yang B, Song J, et al.; China Novel Coronavirus Investigating and Research Team. A novel coronavirus from patients with pneumonia in China, 2019. N Engl J Med. 2020 Feb;382(8):727-33. https://doi. org/10.1056/NEJMoa2001017 PMID:31978945

8. Chen N, Zhou M, Dong $X$, Qu J, Gong F, Han Y, et al. Epidemiological and clinical characteristics of 99 cases of 2019 novel coronavirus pneumonia in Wuhan, China: a descriptive study. Lancet. 2020 Feb;395(10223):507-13. https://doi.org/10.1016/ s0140-6736(20)30211-7 PMID:32007143

9. Lu H, Stratton CW, Tang YW. Outbreak of pneumonia of unknown etiology in Wuhan, China: the mystery and the miracle. J Med Virol. 2020 Apr;92(4):401-2. https:// doi.org/10.1002/jmv.25678 PMID:31950516
10. Bwire GM, Paulo LS.

Coronavirus disease-2019: is

fever an adequate screening for the returning travelers? Trop Med Health. 2020 Mar;48(1):14. https://doi.org/10.1186/s41182020-00201-2 PMID:32165854

11. Lai CC, Shih TP, Ko WC, Tang HJ, Hsueh PR. Severe acute respiratory syndrome coronavirus 2 (SARS-CoV-2) and coronavirus disease-2019 (COVID-19): the epidemic and the challenges. Int J Antimicrob Agents. 2020 Mar;55(3):105924. https://doi.org/10.1016/j. ijantimicag.2020.105924 PMID:32081636

12. Barry M, Al Amri M, Memish ZA. COVID-19 in the Shadows of MERS-CoV in the Kingdom of Saudi Arabia. J Epidemiol Glob Health. 2020 Mar;10(1):1-3. https://doi. org/10.2991/jegh.k.200218.003 PMID:32175703

13. Liu R, Han H, Liu F, Lv Z, Wu $K$, Liu $Y$, et al. Positive rate of RT-PCR detection of SARSCoV-2 infection in 4880 cases from one hospital in Wuhan, China, from Jan to Feb 2020. Clin Chim Acta. 2020 Jun;505:172-5. https://doi. org/10.1016/j.cca.2020.03.009 PMID:32156607

14. Mahase E. Covid-19: WHO declares pandemic because of "alarming levels" of spread, severity, and inaction. BMJ. 2020 Mar;368:m1036. https:// doi.org/10.1136/bmj.m1036 PMID:32165426

15. Velavan TP, Meyer CG. The COVID-19 epidemic. Trop Med Int Health. 2020 Mar;25(3):27880. https://doi.org/10.1111/ tmi.13383 PMID:32052514

16. Wang LS, Wang YR, Ye DW, Liu QQ. A review of the 2019 Novel Coronavirus (COVID-19) based on current evidence.
Int J Antimicrob Agents. 2020 Mar;105948:105948. https://doi.org/10.1016/j. ijantimicag.2020.105948 PMID:32201353

17. Zheng $Y Y, M a Y T$, Zhang JY, Xie X. COVID-19 and the cardiovascular system. Nat Rev Cardiol. 2020 Mar;1-2: https:// doi.org/10.1038/s41569-0200360-5 PMID:32139904

18. Remuzzi A, Remuzzi G. COVID-19 and Italy: what next? Lancet. 2020 Apr;395(10231):1225-8. https://doi.org/10.1016/ S0140-6736(20)30627-9 PMID:32178769

19. Kannan S, Shaik Syed Ali $P$, Sheeza A, Hemalatha K. COVID-19 (Novel Coronavirus 2019) - recent trends. Eur Rev Med Pharmacol Sci. 2020 Feb;24(4):2006-11. https://doi.org/10.26355/ eurrev_202002_20378 PMID:32141569

20. Zhao S, Chen H. Modeling the epidemic dynamics and control of COVID-19 outbreak in China. Quant Biol. 2020 Mar;8(1):1-9. https://doi.org/10.1007/s40484020-0199-0 PMID:32219006

21. Scopus. SciVerse Scopus fact sheet. Sciverse $₫$ Scopus. Amsterdam, Netherlands: Elsevier B.V. 2020. http://www. elsevier.com/online-tools/scopus

22. Lawani SM. Bibliometrics: Its Theoretical Foundations, Methods and Applications. Libri. 1981;31(1):294-315. https://doi. org/10.1515/libr.1981.31.1.294.

23. Subramanyam K. Bibliometric studies of research collaboration: A review. J Inf Sci. 1983;6(1):33-8. https://doi. org/10.1177/016555158300 600105

24. van Eck NJ, Waltman L. Software survey: VOSviewer, a computer program for bibliometric mapping. Scientometrics. 2010 
Aug;84(2):523-38. https://doi. org/10.1007/s11192-009-01463 PMID:20585380

25. Huang $C$, Wang $Y$, Li $X$, Ren L, Zhao J, Hu $Y$, et al. Clinical features of patients infected with 2019 novel coronavirus in Wuhan, China. Lancet. 2020 Feb;395(10223):497-506. https://doi.org/10.1016/ S0140-6736(20)30183-5 PMID:31986264

26. Chan JF, Yuan S, Kok KH, To $\mathrm{KK}, \mathrm{Chu} \mathrm{H}$, Yang J, et al. A familial cluster of pneumonia associated with the 2019 novel coronavirus indicating personto-person transmission: a study of a family cluster. Lancet. 2020 Feb;395(10223):514-23. https://doi.org/10.1016/
S0140-6736(20)30154-9

PMID:31986261

27. Wang $D, H u$ B, Hu C, Zhu F, Liu X, Zhang J, et al. Clinical Characteristics of 138 Hospitalized Patients With 2019 Novel CoronavirusInfected Pneumonia in Wuhan, China. JAMA. 2020 Feb;323(11):1061. https://doi org/10.1001/jama.2020.1585 PMID:32031570

28. Holshue ML, DeBolt C, Lindquist S, Lofy KH, Wiesman J, Bruce $\mathrm{H}$, et al.; Washington State 2019-nCoV Case Investigation Team. First case of 2019 novel coronavirus in the United States. N Engl J Med. 2020 Mar;382(10):929-36. https://doi. org/10.1056/NEJMoa2001191
PMID:32004427

29. Rothe $C$, Schunk $M$, Sothmann P, Bretzel G, Froeschl G, Wallrauch $C$, et al. Transmission of 2019-NCOV infection from an asymptomatic contact in Germany. N Engl J Med. 2020 Mar;382(10):970-1. https://doi. org/10.1056/NEJMc2001468 PMID:32003551

30. Hui DS, I Azhar E, Madani TA, Ntoumi F, Kock R, Dar O, et al. The continuing 2019$\mathrm{nCoV}$ epidemic threat of novel coronaviruses to global health - The latest 2019 novel coronavirus outbreak in Wuhan, China. Int J Infect Dis. 2020 Feb;91:264-6. https://doi. org/10.1016/j.ijid.2020.01.009 PMID:31953166 